\title{
Elemental Source Composition Measurements and the Origin of Galactic Cosmic Rays - ACE-CRIS Observations of UH Elements
}

\author{
W. R. Binns \\ Washington University in St. Louis \\ St. Louis, MO, USA \\ wrb@wustl.edu \\ M. H. Israel ${ }^{\mathbf{1}}$ \\ Washington University in St. Louis \\ St. Louis, MO, USA \\ mhi@wustl.edu
}

M. E. Wiedenbeck

Jet Propulsion Lab, California Institute of Technology

Pasadena, CA, USA

mark.e.wiedenbeck@jpl.nasa.gov

\author{
A. C. Cummings, R. A. Leske, R. A Mewaldt, E. C. Stone \\ California Institute of Technology \\ Pasadena, CA, USA \\ ace@srl.caltech.edu,ral@srl.caltech.edu,rmewaldt@srl.caltech.edu,ecs@srl.caltech.edu,
}

\section{E. R. Christian, G. A. de Nolfo, T. T. von Rosenvinge}

NASA Goddard Space Flight Center

Greenbelt, MD, USA

eric.r.christian@nasa.gov,georgia.a.denolfo@nasa.gov,tycho@starpower.net

\begin{abstract}
We present measurements of the elemental abundances of ultra-heavy ( $\mathrm{Z}>29)$ cosmic rays made by the CRIS instrument on NASA's ACE satellite. The data set corresponds to 7406 days of data collection from December 4, 1997 through Feb. 18, 2019. We detected a total of 1205 ultra-heavy nuclei in the charge range of $\mathrm{Z}=30-40$. The charge resolution that we obtain shows essentially complete separation of the elements over this charge range. We derive preliminary source abundances from these measurements. These source abundances show that the ordering of refractory and volatile elements with atomic mass is greatly improved when compared to a mix of massive star outflow plus SN ejecta with normal ISM, rather than with only normal ISM. Additionally, in this ordering the refractory and volatile elements have similar slopes and refractory elements are preferentially accelerated by a factor of $\sim 4$. The measured abundances support a model in which cosmic ray source material is a mix of massive star outflow plus supernova ejecta and normal ISM, consistent with conclusions reached from the SuperTIGER high-altitude balloon measurements.
\end{abstract}




\section{Introduction}

The Cosmic Ray Isotope Spectrometer (CRIS) [1] on the Advanced Cosmic Explorer (ACE) spacecraft [2] has been in halo orbit about the L1 Lagrange point for nearly 22 years. It is still today returning excellent data on the elemental and isotopic composition of Galactic Cosmic Rays (GCR). With its geometrical factor $250 \mathrm{~cm}^{2} \mathrm{sr}$, and its originally expected life-time of 2 to 5 years, the primary objective of CRIS was focussed on nuclei between ${ }_{4} \mathrm{Be}$ and ${ }_{28} \mathrm{Ni}$ that stop in a stack of silicon solid-state detectors [1]. The UltraHeavy (UH) elements, those with atomic number, $\mathrm{Z}>29$ are much rarer in the GCR than the lighter elements, with the abundance of ${ }_{30} \mathrm{Zn}$ $\sim 10^{-4}$ times that of ${ }_{26} \mathrm{Fe}$, and each of the elements with $\mathrm{Z}>30$ having at most $\sim 10^{-5}$ times that of ${ }_{26} \mathrm{Fe}$. With the very long life of CRIS, we are now able to report abundances of elements with $\mathrm{Z}$ as high as 40 .

\section{Observations of UH elements in the GCR}

Figure 1 displays data gathered by CRIS over the period from 1997 December 4 through 2019 February 18, a total of 7406 days of actual data. This data set corresponds to $1.5 \times 10^{6}{ }_{26} \mathrm{Fe}$ nuclei. We see excellent charge resolution for all elements up to ${ }_{40} \mathrm{Zr}$. The various element peaks have various widths, primarily the result of the number of stable isotopes for each element. The red and blue lines show the calculated positions of stable isotopes for an element, and the red and blue circles show the calculated positions of isotopes that can only decay by electron-capture and thus are stable when fully stripped. (Red lines and circles are for even-Z elements, and blue for odd-Z elements.)

Figure 2 displays the same data set as the previous figure, but with finer binning, indicating clear isotope resolution for elements up to ${ }_{32} \mathrm{Ge}$ and beyond.

Analysis of these isotope data is still in progress, and this paper confines itself to the element analysis.

\section{Derivation of In-Space abundances}

To derive relative element abundances in space from the CRIS observations

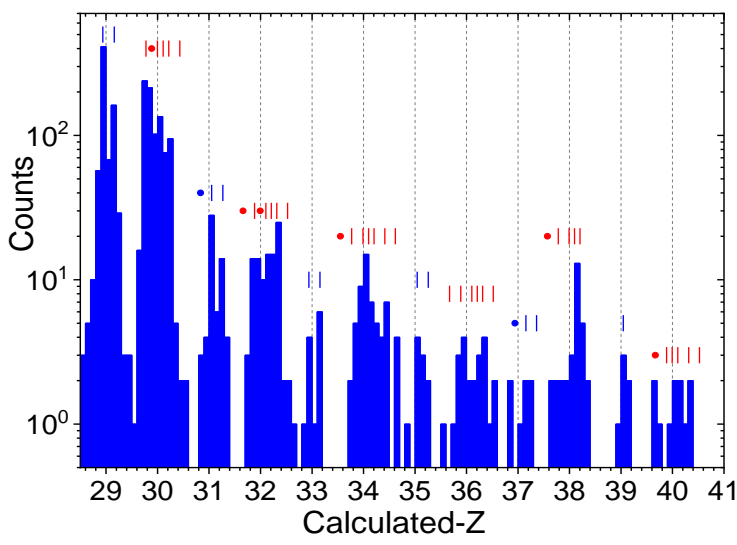

Fig. 1-Histogram of cosmic rays with $Z=29-41$.

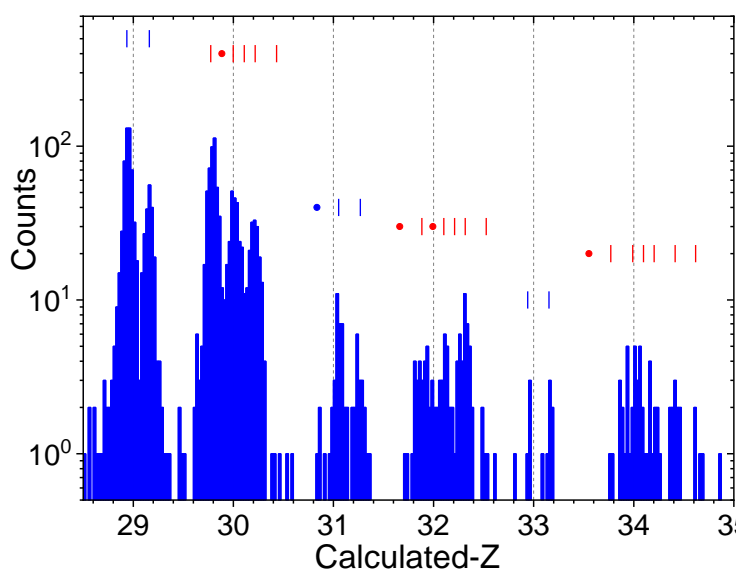

Fig. 2-Same data as Fig. 1 with finer binning. requires application of three correction factors, which are displayed in Figure 3: Correction for loss of events due to nuclear interactions in the CRIS detector, correction for the Z-dependent width of the energy band 
recorded in CRIS as well as the energy spectrum shape in the band for each element, and correction for loss of events due to saturation in the pulse-height analyzers (PHA).

Incident nuclei that suffer nuclear interactions in the CRIS instrument give inconsistent measurements in the various detectors of the instrument and are eliminated from the analysis. Since heavier nuclei have larger interaction cross-sections than lighter nuclei, the interactioncorrection factor increases with $\mathrm{Z}$.

CRIS data displayed in Figure 1 are for nuclei that stop in the silicon detector stack, so the width of the energy band increases with $\mathrm{Z}$. The band is $120-570 \mathrm{MeV} /$ nucleon for ${ }_{26} \mathrm{Fe}$, growing to $150-730$

$\mathrm{MeV} /$ nucleon for ${ }_{40} \mathrm{Zr}$ and furthermore the cosmic-ray energy spectrum is somewhat different in the band for each element.

The largest correction factor results from elimination of events in which the data showed PHA saturation. Since the primary objective of CRIS was measurement of elements with $3<\mathrm{Z}<30$, the instrumentation was designed with a dynamic range to cover all possible signals from nuclei in this interval. For nuclei with Z> 32, the widest-angle, lowestenergy incident cosmic rays saturated at least some of the PHAs. For higher $\mathrm{Z}$ the range of saturating angles and energies increased, resulting in a saturation-correction factor that increases steeply with Z. The

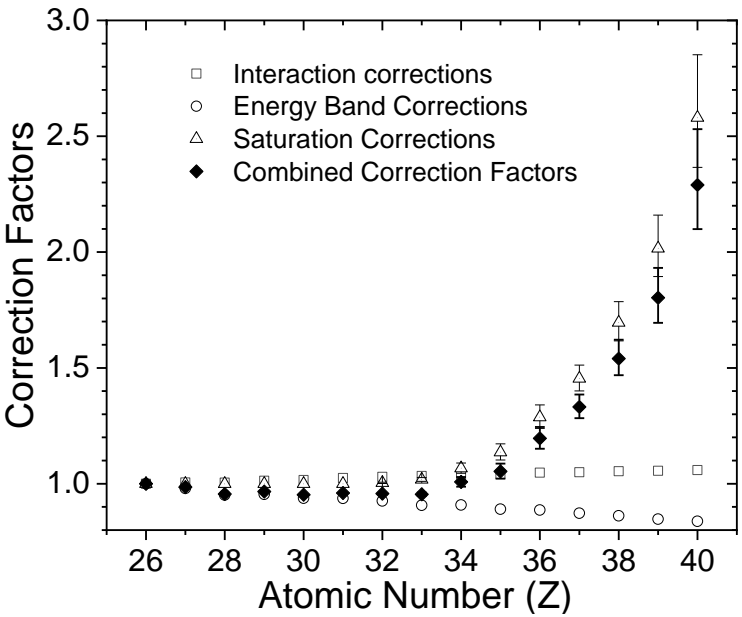

Fig. 3-Correction factors for interactions in the instrument, differing energy bands, PHA saturation, and combined.

saturation corrections plotted in Figure 3 are derived from an extensive Monte Carlo calculation. The plotted uncertainties reflect worst-case variations in gains and thicknesses among the detectors. The factors are quite substantial for $\mathrm{Z} \geq 38$, but we believe they are well understood.

\section{Comparison of CRIS results with those from SuperTIGER}

Figure 4 compares the in-space relative abundances derived from the CRIS data with abundances, extrapolated to the top of the atmosphere, from the balloon-borne SuperTIGER (ST) [3]. We note that the error bars on the ST abundances are smaller than those on CRIS, 
primarily because the ST data set corresponds to $4.2 \times 10^{6}{ }_{26} \mathrm{Fe}$ nuclei, nearly three times larger than the CRIS data set. The ST data were from a 55-day high-altitude balloon flight over Antarctica in December 2012 - January 2013.

We note the good, but not perfect agreement between these two sets of in-space abundances. There are several differences in the conditions under which these two sets of data were gathered, some of which may be responsible for the differences in abundances.

Perhaps the most significant difference between the two data sets is the energy interval. CRIS measured abundances of nuclei stopping in the silicon detector stack, energies between 125 and $725 \mathrm{MeV} /$ nucleon. ST measured particles with energy inside the instrument above the Plexiglas Cherenkov detector threshold of $325 \mathrm{MeV} /$ nucleon. When extrapolated to the top of the instrument and then to the top of the atmosphere, this lower-energy limit corresponds to approximately $800 \mathrm{MeV} /$ nucleon. So ST measured abundances for elements with all energies in space greater than $0.8 \mathrm{GeV} /$ nucleon.

The CRIS data were gathered over more than 21 years, covering two complete cycles of solar modulation, while ST data were gathered over less than two months at an intermediate level of solar modulation characterized by $\phi \sim 540 \mathrm{MV}$. Also the CRIS data were gathered in space, while the ST data were gathered under about $4 \mathrm{mb}$ of atmosphere and thus needed to be extrapolated to the top of the atmosphere.

Figure 5 compares the CRIS and ST results when extrapolated back to the GCR source (GCRS), taking account of interactions and

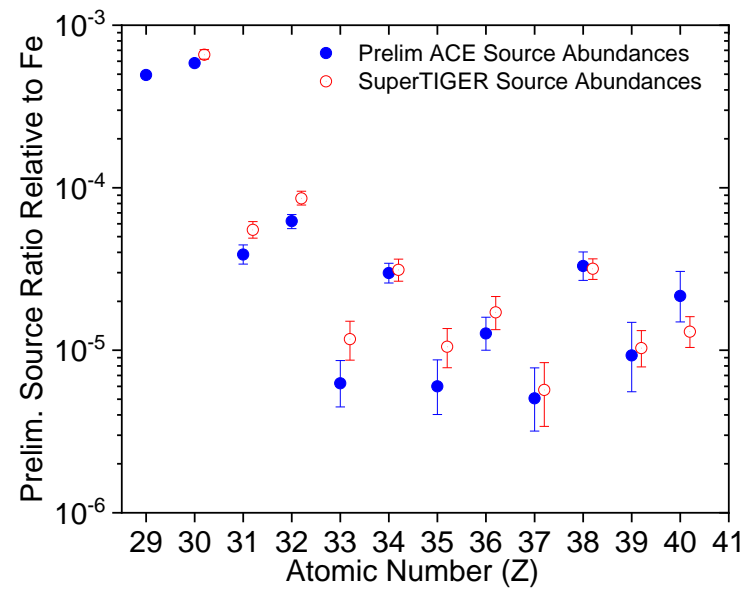

Fig. 5-Preliminary CRIS elemental source abundances compared with ST source abundances. energy loss while propagating through the interstellar medium as well as energy loss while undergoing solar modulation. The small differences for $\mathrm{Z}$ in the range $30-33$ between the two sets of results are mainly reflecting the differences in the "in-space" observations. Those differences could be due in part to the
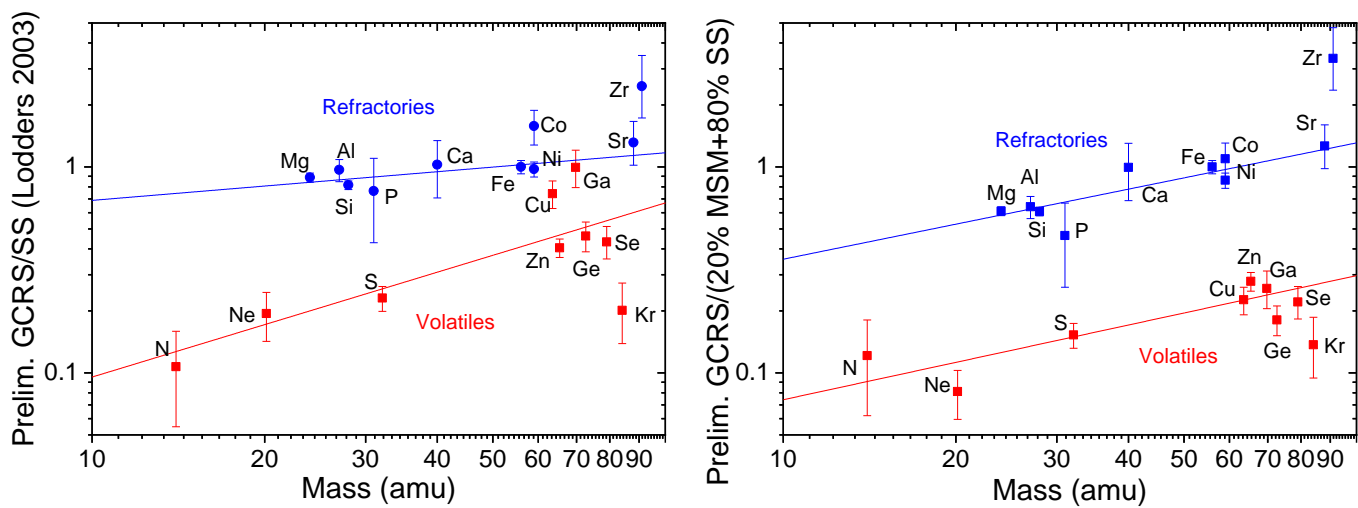

Fig. 6-Plots of earlier preliminary CRIS data plotted relative to SS abundances (left panel) and a mix of $20 \%$ massive star material and $80 \%$ normal ISM (right panel). 
differences between the energies to which the two instruments were sensitive, and could possibly be due in part to contamination of the low 30s in ST by non-Gaussian tails from Fe and Ni.

\section{Conclusions about GCR origins}

Figure 6 is very similar to one displayed previously by Murphy et al. [3]. As was shown there, when one plots as a function of mass the GCRS abundances relative to a mix of massive star outflow plus SN ejecta with normal Inter-Stellar Material (ISM) instead of simply relative to normal ISM (taken as the Lodders [4] solar-system abundances), the refractories and volatile elements separate nicely. This substantially improved ordering of the GCR abundances when taken relative to a mix instead of just normal ISM argues

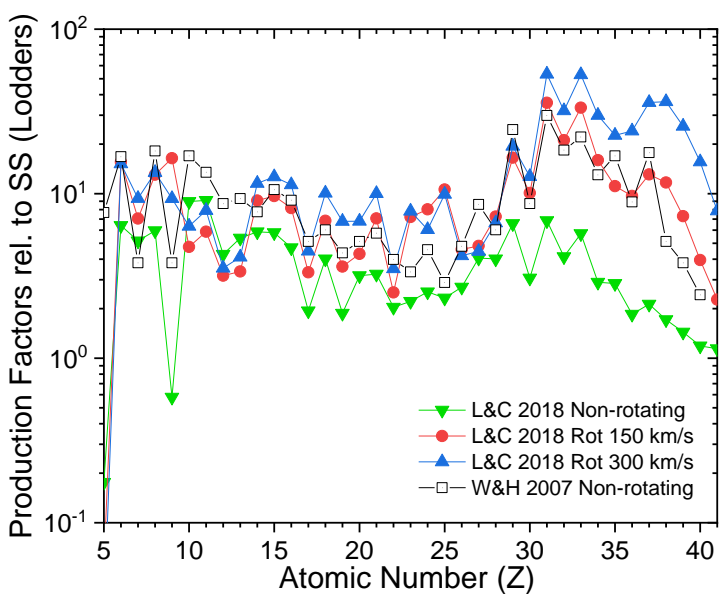

Fig. 7-Production factors of elements relative to SS taken from Limongi \& Chieffi [6]. that the GCRs are accelerated from a mix of massive star material and ISM, as would be found in an $\mathrm{OB}$ association.

However, the fraction of massive star material mixing with normal ISM is clearly dependent upon the model of stellar production of heavy nuclei used. In figure 6 we used the heavy nuclei production from the work of Woosley \& Heger $(\mathrm{W} \& \mathrm{H})$ [5]. More recently, Limongi \& Chieffi $(\mathrm{L} \& \mathrm{C})[6]$ have calculated production yields for both non-rotating massive-star models and for models with various speeds of rotation. In Figure 7 we see that L\&C non-rotating model produces much less of heavy nuclei than the $\mathrm{W} \& \mathrm{H}$ model, which is also non-rotating. The L\&C $150 \mathrm{~km} / \mathrm{s}$ rotating model produces about the same abundance of heavy nuclei as the $\mathrm{W} \& \mathrm{H}$ model, while the L\&C $300 \mathrm{~km} / \mathrm{s}$ rotating model produces more heavy nuclei.

Thus firm conclusions about GCR origin must await improved models of production of heavy nuclei (e.g. [7]). Recent advances in 3D modeling of supernovae promise improved estimates of abundances of heavy nuclei production.

This research is supported by NASA under Grant \# 80NSSC18K0223

\section{References}

[1] E. C. Stone, C. M. S. Cohen, W. R. Cook, et al., The Cosmic-Ray Isotope Spectrometer for the Advanced Composition Explorer, Space Science Reviews 86, 285 (1998). 
[2] E. C. Stone, A. M. Fransden, R. A. Mewaldt, et al., The Advanced Composition Explorer, Space Science Reviews 86, 1 (1998).

[3] R. P. Murphy, M. Sasaki, W. R. Binns, et al., Galactic Cosmic Ray Origins and OB Associations: Evidence From SuperTIGER Observations of Elements ${ }_{26} \mathrm{Fe}$ through ${ }_{40} \mathrm{Zr}$, Astrophysical Journal 831, 148 (2016).

[4] K. Lodders, Solar System Abundances and Condensation Temperatures of the Elements, Astrophysical Journal 591, 1220 (2003).

[5] S. E. Woosley \& A. Heger, Nucleosyntheses and Remnants in Massive Stars of Solar Metallicity, Physics Reports 442, 269 (2007).

[6] M. Limongi \& A. Chieffi, Presupernova Evolution and Explosive Nucleosynthesis of Rotating Massive Stars in the Metallicity range $-3 \leq[\mathrm{Fe} / \mathrm{H}] \leq 0$. Astrophysical Supplement Series 237, 13 (2018).

[7] D. Vartanyan, A. Burrows, \& D. Radice, Temporal and Angular Variations of 3D Core-Collapse Supernova Emissions and their Physical Correlations, astro-ph>arXiv:1906.08787 (2019). 